\title{
Realidade virtual como recurso fisioterapêutico no tratamento cardiorrespiratório:
}

\section{Uma revisão sistemática}

\author{
Virtual reality as a physical therapy resource in cardiorespiratory treatment: A systematic review \\ La realidad virtual como recurso fisioterapéutico en el tratamiento cardiorrespiratorio: Una
}

revisión sistemática

Recebido: 14/12/2021 | Revisado: 16/12/2021 | Aceito: 23/12/2021 | Publicado: 25/12/2021

\author{
Elaine Becher Santos \\ ORCID: https://orcid.org/0000-0002-2088-059X \\ Hospital Universitário Regional de Ponta Grossa, Brasil \\ E-mail: elainebsantos06@gmail.com \\ Talita Wassmutch \\ ORCID: https://orcid.org/0000-0002-2816-2867 \\ Hospital Universitário Regional de Ponta Grossa, Brasil \\ E-mail: taliwassmuth2015@outlook.com \\ Juliana Carvalho Schleder \\ ORCID: https://orcid.org/0000-0001-5789-7945 \\ Hospital Universitário Regional de Ponta Grossa, Brasil \\ E-mail: juschleder@yahoo.com.br \\ Debora Melo Mazzo \\ ORCID: https://orcid.org/0000-0002-1728-7648 \\ Hospital Universitário Regional de Ponta Grossa, Brasi \\ E-mail: debora.mazzo@uepg.br
}

\begin{abstract}
Resumo
O objetivo desta revisão sistemática foi verificar as principais evidências descritas na literatura relacionadas ao uso da realidade virtual (RV) no tratamento fisioterapêutico cardiorrespiratório. A busca dos estudos foi realizada nas bases de dados LILACS, PubMed, PEDro, Scielo e Scopus. Foram selecionados estudos originais que abordaram o uso da RV no tratamento fisioterapêutico cardiorrespiratório em idiomas português, inglês e espanhol. Durante a pesquisa, foram encontrados 415 estudo dos quais, cinco estudos contemplaram os critérios de inclusão. Os estudos foram submetidos a julgamento do risco de viés através da ferramenta Cochrane Risk of Bias Tool. Dos cinco estudos selecionados, nenhum apresentou alto risco de viés. Quatros estudos utilizaram à RV não imersiva como recurso auxiliar. Três estudos foram realizados em ambiente hospitalar e dois em ambulatorial. Dois estudos avaliaram a capacidade funcional através da MIF e três estudos utilizaram o TC6 para avaliar a capacidade cardiorrespiratória. Apenas um estudo avaliou os efeitos hemodinâmicos agudos produzido pelo uso da RV na terapia e outro a função pulmonar através da avaliação das forças inspiratórias e expiratórias máximas. Todos os atendimentos de RV eram associados a cinesioterapia. Concluiu-se que o uso da RV como método coadjuvante no tratamento fisioterapêutico cardiorrespiratório, tanto em ambiente hospitalar quanto ambulatorial, apresenta resultados semelhantes ao tratamento convencional na melhora da capacidade funcional e física, com benefícios motores e respiratórios.
\end{abstract}

Palavras-chave: Realidade virtual; Terapia de exposição à realidade virtual; Reabilitação; Fisioterapia.

\begin{abstract}
This systematic review aimed to verify on main literature evidence related to virtual reality (VR) used for cardiorespiratory physical therapy. The search for studies was performed in LILACS, PubMed, PEDro, Scielo and Scopus databases. Original studies that addressed use of VR in cardiorespiratory physical therapy treatment in Portuguese, English and Spanish were selected. During the search, four hundred fifteen studies were found, of which five studies met the inclusion criteria. The studies were submitted to risk of bias judgment using the Cochrane Risk of Bias Tool. From five studies selected, none showed a high risk of bias. Four studies used non-immersive VR as an auxiliary resource. Three studies were conducted in inpatient and two in outpatient settings. Two studies assessed functional capacity using FIM and three studies used 6MWT to assess cardiorespiratory capacity. Only one study evaluated acute hemodynamic effects produced by VR in therapy and another evaluated pulmonary function through the evaluation of maximal inspiratory and expiratory forces. All VR treatments were associated with kinesiotherapy. It was concluded that VR as a coadjuvant method in cardiorespiratory physiotherapy treatment, both in hospital and outpatient settings, presents results similar to conventional treatment in improving functional and physical capacity, with motor and respiratory benefits.
\end{abstract}

Keywords: Virtual reality; Virtual reality exposure therapy; Rehabilitation; Physical therapy. 


\begin{abstract}
Resumen
El objetivo de esta revisión sistemática fue verificar las principales evidencias descritas en la literatura relacionadas con el uso de la realidad virtual (RV) en el tratamiento de fisioterapia cardiorrespiratoria. La búsqueda de estudios se realizó en las bases de datos LILACS, PubMed, PEDro, Scielo y Scopus. Se seleccionaron estudios originales que abordaban el uso de la RV en el tratamiento de fisioterapia cardiorrespiratoria en portugués, inglés y español. Durante la búsqueda, se encontraron 415 estudios, de los cuales cinco cumplían los criterios de inclusión. Los estudios se sometieron al juicio del riesgo de sesgo a través de la Herramienta Cochrane de Riesgo de Sesgo. De los cinco estudios seleccionados, ninguno presentó un alto riesgo de sesgo. Cuatro estudios utilizaron la RV no inmersiva como recurso auxiliar. Tres estudios se llevaron a cabo en pacientes internos y dos en pacientes externos. Dos estudios evaluaron la capacidad funcional mediante el FIM y tres estudios utilizaron la 6MWT para evaluar la capacidad cardiorrespiratoria. Sólo un estudio evaluó los efectos hemodinámicos agudos producidos por el uso de la RV en la terapia y otro evaluó la función pulmonar mediante la valoración de las fuerzas inspiratorias y espiratorias máximas. Todos los servicios de RV estaban asociados a la kinesioterapia. Se concluyó que el uso de la RV como método coadyuvante en el tratamiento de fisioterapia cardiorrespiratoria, tanto en el ámbito hospitalario como en el ambulatorio, presenta resultados similares al tratamiento convencional en la mejora de la capacidad funcional y física, con beneficios motores y respiratorios.
\end{abstract}

Palabras clave: Realidad virtual; Terapia de exposición a la realidad virtual; Rehabilitación; Terapia física.

\title{
1. Introdução
}

A fisioterapia tem sua origem atrelada ao processo de reabilitação de inúmeras disfunções motoras, respiratórias e funcionais (Oliveira, Souza, Coutinho, Castro, \& Paulo, 2015). Atua com diversas especialidades e uma em particular, a fisioterapia cardiorrespiratória, apresenta os cuidados voltados ao sistema cardiovascular e pulmonar, que avalia o comprometimento físico e funcional resultantes de alterações geradas por uma doença, lesão ou outra condição nesses sistemas (Okasheh et al., 2019).

O tratamento fisioterapêutico cardiorrespiratório baseia-se, principalmente na prescrição clínica de exercícios, e no uso de manobras e técnicas específicas, resultando na estabilização ou reversão das manifestações agudas e crônicas das doenças (Almeida \& Rodrigues, 2014; Bernard et al., 2014; Carvalho, 2012). Para atingir estes resultados, a fisioterapia dispõe de recursos como, a terapia manual, mecanoterapia, cinesioterapia, agentes físicos, entre outros Tanović et al. (2019).

Com a expansão tecnológica mundial, diversas áreas da saúde começaram a difundir em seu meio a utilização de recursos mais avançados, voltados para o mundo digital, e não poderia ser diferente com a fisioterapia, que atualmente utiliza a realidade virtual (RV) como ferramenta adicional para os tratamentos propostos (Caiana et al., 2016), visando viabilidade, adaptabilidade, estimulação motivacional, resultando em benefícios positivos na recuperação (Burdea, 2003).

A RV é uma importante aliada para os fisioterapeutas no quesito de recurso complementar, pois além de fornecer ao paciente estímulo sensório-motor, motiva a sua interação com a terapia e quando aplicado ao movimento, apresenta melhora da funcionalidade (Rutkowski et al., 2020). Entretanto, não há estudos restritos ao uso da RV na reabilitação cardiorrespiratória, apresentando poucos relatos na área. Considerando o exposto, este estudo teve como objetivo sintetizar as principais evidências descritas na literatura relacionadas ao uso da RV na fisioterapia cardiorrespiratória.

\section{Metodologia}

Para a realização desta revisão sistemática, a pesquisa bibliográfica foi feita por dois revisores distintos, que utilizaram como pergunta norteadora: “Quais são as principais evidências sobre o uso da RV na reabilitação cardiorrespiratória e seus benefícios dentro de um atendimento fisioterapêutico?". As buscas dos artigos foram baseadas no modelo Populations, Interventions, Comparison, Outcome (PICO), recomendado para pesquisas de revisão bibliográfica e instrumento para práticas clínicas baseadas em evidências (Santos et al., 2007).

Esse modelo de pesquisa tem como característica o desenvolvimento de perguntas objetivas, utilizando métodos sistematizados e claros, com o foco de identificar, selecionar e avaliar criteriosamente estudos relevantes e de qualidade 
metodológica (Galvão et al., 2015). Assim, optou-se por utilizar a recomendação PRISMA, que apresenta um checklist com 27 itens e um fluxograma.

Foi realizada a busca nas bases de dados LILACS, Medline (PubMed), PEDro, Scielo e Scopus, no período de dezembro de 2020 a fevereiro de 2021. Para a busca foram utilizados os descritores disponíveis no DeCS (Descritores em Ciências da Saúde) em inglês: Virtual Reality; Virtual Reality Exposure Therapy; Rehabilitation; Physiotherapy. Os cruzamentos desses descritores foram realizados utilizando o boleador AND.

Os critérios de inclusão foram ensaios clínicos randomizados que utilizassem à RV como método coadjuvante ou alternativo na recuperação física e funcional dentro da fisioterapia, nas áreas cardiovascular e pulmonar, apresentados disponível na íntegra, publicados em português, inglês e espanhol, indexados nos últimos dez anos. Foram excluídos estudos, de intervenções que utilizaram à RV no desenvolvimento de protocolos de criação de jogos de computador, que utilizaram à RV como meio de avaliação, artigos duplicados, com metodologias qualitativas e de viabilidade, e que não apresentassem no título, no resumo, ou no texto o tema abordado.

A identificação dos estudos foi realizada em três etapas: (1) Leitura dos títulos dos estudos e exclusão dos que não se enquadraram nos critérios de inclusão estabelecidos; (2) Leitura e análise dos resumos dos estudos selecionados na primeira etapa e exclusão dos artigos que não apresentaram critérios estabelecidos; (3) Leitura e análise na íntegra de todos os estudos restantes e seleção dos que se enquadraram na pesquisa.

Para análise do risco de viés, foram utilizados os critérios descritos no Capítulo 8 do Manual Cochrane para revisões sistemáticas de intervenções (Carvalho \& Silva, 2013) considerando os domínios, geração de sequência aleatória, ocultação de alocação, cegamento de participantes e pessoal, cegamento dos avaliadores de desfechos, desfechos incompletos, relatórios de desfechos seletivos, e outras fontes de viés.

Cada estudo teve sua fonte potencial de viés julgada como alta (vermelho), baixa (verde) ou incerta (amarela). Além disso, os dados dos artigos foram analisados detalhadamente, considerando os pontos, citação, objetivos, participantes, variáveis analisadas, tipo de RV utilizada, protocolo de RV adotado, principais resultados, e eventos adversos.

\section{Resultados}

Foram encontrados 415 estudos nas bases de dados, sendo 60 encontrados na Scielo, 217 na PubMed, 37 no PEDro, 6 na LILACS e 95 na Scopus. Após a aplicação dos critérios de elegibilidade, foram selecionados cinco artigos para a realização desta revisão (Figura 1). 
Figura 1 - Diagrama de fluxo da seleção do estudo.

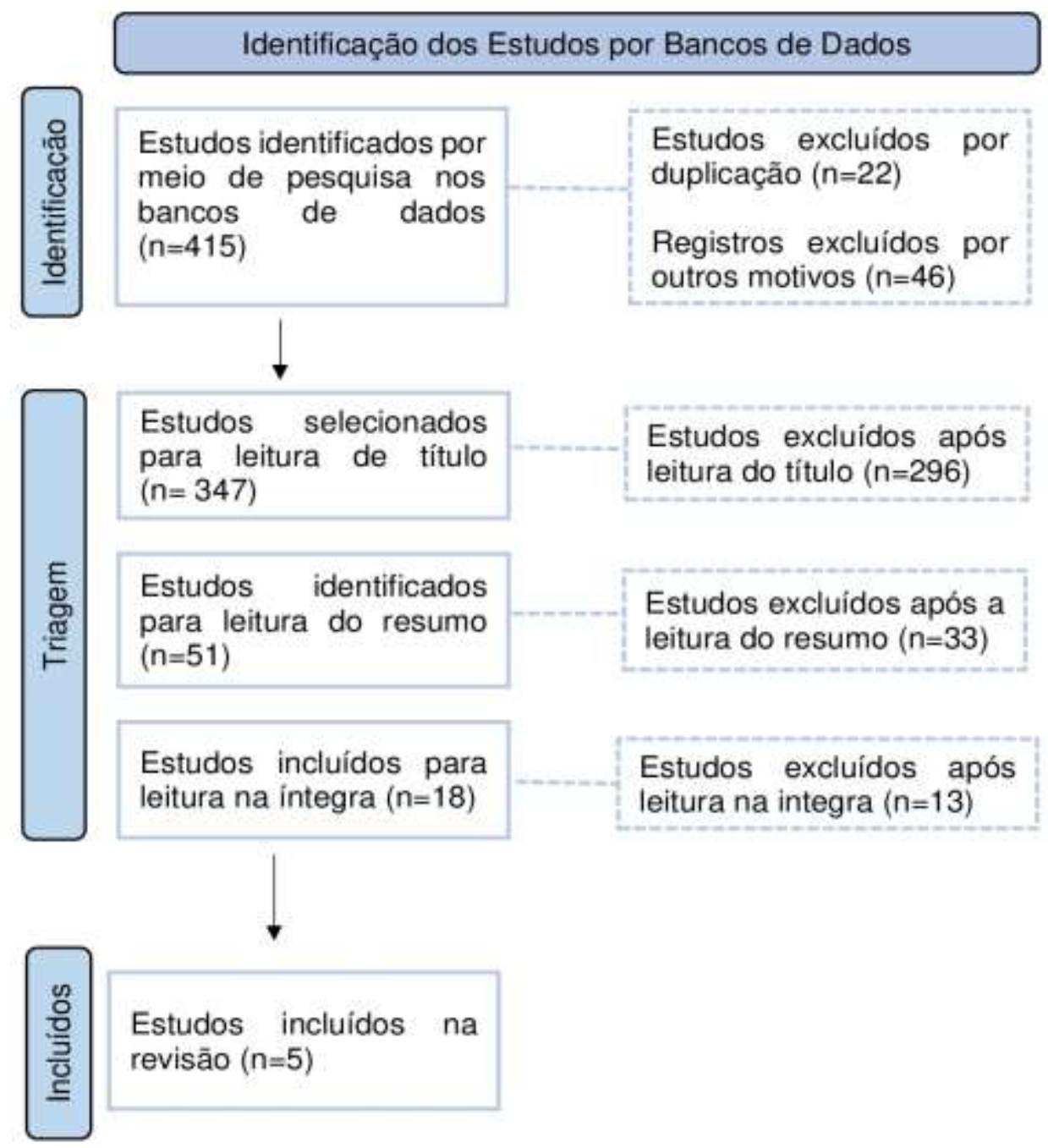

Fonte: Page, M. J. et al.(2021). A declaração PRISMA 2020: uma diretriz atualizada para relatar revisões sistemáticas. Bmj, 372.

As datas de publicação dos estudos analisados foram dos últimos 10 anos, sendo incluídas pesquisas brasileiras e internacionais. A amostragem foi outro fator relevante, os cinco ensaios clínicos randomizados envolveram um total de 280 números de participantes. A população dos estudos foi composta por pacientes com idade média de 58 anos, que apresentavam alteração cardíaca ou pulmonar aguda ou crônica, e que foram submetidos a atendimento fisioterapêutico. Os detalhes sobre os potenciais risco de viés estão resumidos na Figura 2. 
Figura 2 - Resumo do risco de viés: análise dos julgamentos dos autores sobre cada item de risco de viés para cada estudo incluído
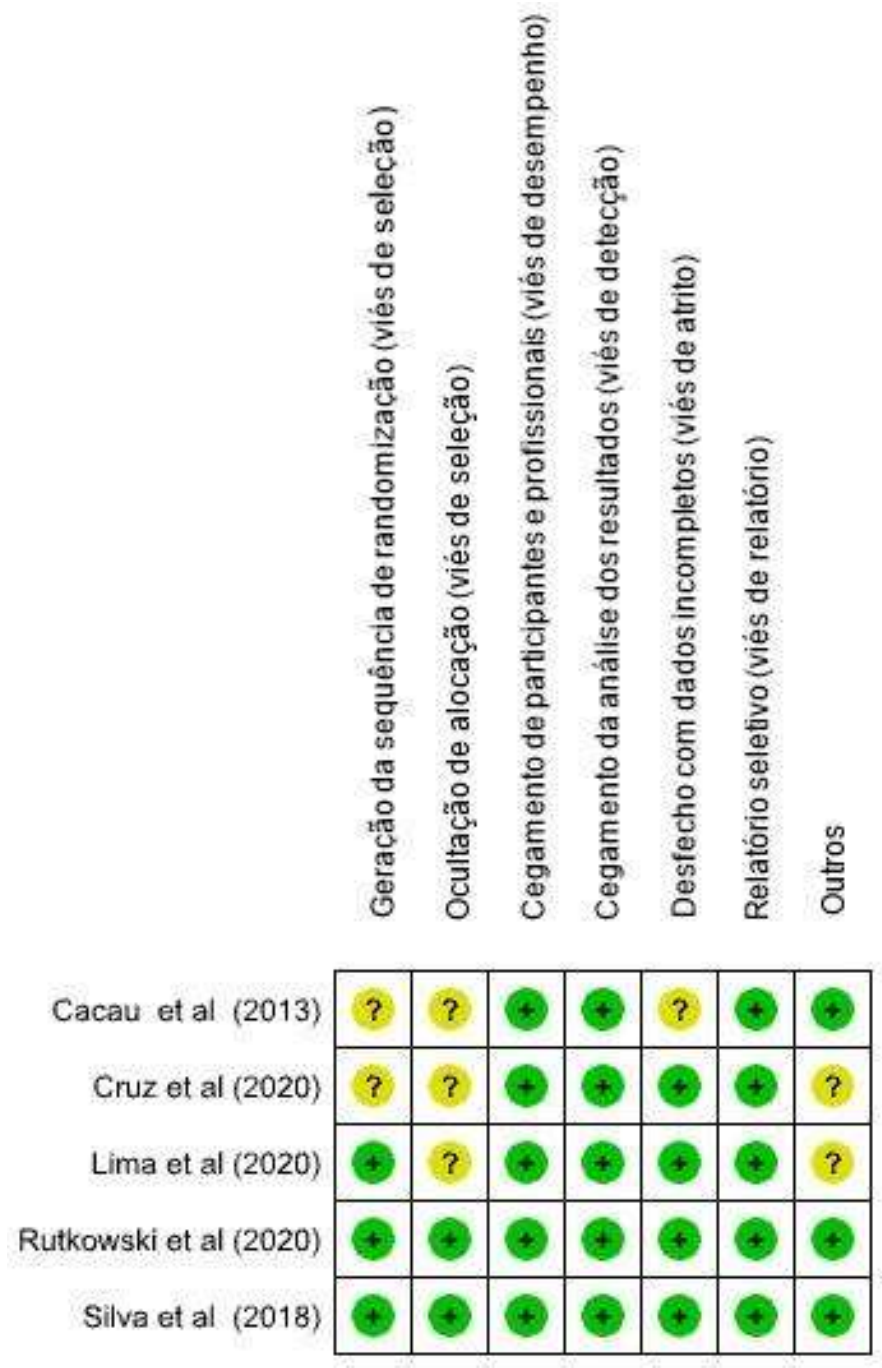

Fonte: Adaptado do sistema Review Manager 5.4.

$\mathrm{Na}$ análise de viés de desempenho e detecção, no qual julga se os estudos realizaram o cegamento de participantes, avaliadores e de resultados, nenhum dos cinco estudos selecionados, todos relataram os resultados conforme planejado, sendo julgados com baixo risco de viés. Dentro do viés de seleção, três estudos (Cacau et al. 2013; Alves da Cruz et al. 2020 e Lima et al. 2020) possuíam informações insuficientes referentes a forma de randomização e ocultação de alocação. Em relação ao viés de Tabela 1. 
Tabela 1 - Principais características dos estudos incluídos.

\begin{tabular}{|c|c|c|c|c|c|c|c|}
\hline Citação & Objetivo & Participantes & $\begin{array}{c}\text { Variáveis } \\
\text { analisadas }\end{array}$ & $\begin{array}{c}\text { Tipo de } \\
\text { RV } \\
\text { utilizada }\end{array}$ & $\begin{array}{l}\text { Protocolo } \\
\text { de RV } \\
\text { adotado }\end{array}$ & Principais resultados & $\begin{array}{c}\text { Eventos } \\
\text { adversos }\end{array}$ \\
\hline $\begin{array}{l}\text { Silva et } \\
\text { al., } 2018\end{array}$ & $\begin{array}{l}\text { Comparar os efeitos da } \\
\text { reabilitação } \\
\text { convencional cardíaca } \\
\text { realidade v virtual na } \\
\text { composição corporal e } \\
\text { capacidade funcional de } \\
\text { pacientes com doenças } \\
\text { cardíacas. }\end{array}$ & $\begin{array}{c}\mathrm{n}=27 \\
\mathrm{GC} \mathrm{n}=13 \\
(\mathrm{~F}=6 / \mathrm{M}=6) \\
\text { GRV } \mathrm{n}=14(\mathrm{~F}=12 / \\
\mathrm{M}=2) \\
\text { Média de idade: } \\
\text { 63,46 anos }\end{array}$ & $\begin{array}{c}\text { Bioimpedância, } \\
\text { TC6, Glicemia } \\
\text { capilar pós } \\
\text { prandial }\end{array}$ & $\begin{array}{c}\text { Xbox } \\
360^{\circledR} \\
\text { Kinect }^{\circledR}\end{array}$ & $\begin{array}{l}60 \mathrm{~min} \\
2 \mathrm{x} / \text { semana } \\
8 \text { semanas } \\
\text { Ambulatorial }\end{array}$ & $\begin{array}{l}\text { A capacidade funcional e o índice } \\
\text { glicêmico de ambos os grupos } \\
\text { melhoraram significativamente } \\
(\mathrm{p}<0,05) \text { após o protocolo de } \\
\text { intervenção, porém quando } \\
\text { comparado um grupo ao outro não } \\
\text { houve diferença entre eles (p>0,05). } \\
\text { A composição corporal do grupo } \\
\text { GRV apresentou alteração } \\
\text { significativa comparado ao grupo } \\
\text { GC }(p>0,05) \text {. }\end{array}$ & $\begin{array}{l}\text { Dispneia } \\
\text { e } \\
\text { cansaço } \\
\text { intenso } \\
(\mathrm{n}=1)\end{array}$ \\
\hline $\begin{array}{l}\text { Cruz et } \\
\text { al., } 2020\end{array}$ & $\begin{array}{l}\text { Analisar os efeitos } \\
\text { hemodinâmicos agudos da } \\
\text { adição de terapia baseada } \\
\text { em } R V \text {, usando exergames } \\
\text { para pacientes submetidos } \\
\text { a reabilitação cardíaca. }\end{array}$ & $\begin{array}{c}\mathrm{n}=27 \\
(\mathrm{M}=14 / \mathrm{F}=13) \\
\text { Média de idade: } \\
63,40 \text { anos }\end{array}$ & $\begin{array}{l}\text { PAS, PAD, } \\
\mathrm{FC}, \mathrm{FR}, \mathrm{FCR}, \\
\mathrm{SpO}_{2}\end{array}$ & $\begin{array}{c}\text { Xbox } \\
360^{\circledR} \\
\text { Kinect }^{\circledR}\end{array}$ & $\begin{array}{l}85 \mathrm{~min} \\
\text { Ambulatorial }\end{array}$ & $\begin{array}{l}\text { Na análise das variáveis de FC, FR, } \\
\text { PAS, PAD } \mathrm{SPO}_{2} \text {, o grupo de GRV } \\
\text { apresentou respostas semelhantes } \\
(\mathrm{p}<0,05) \text { de alterações } \\
\text { hemodinâmicas, atingindo o limiar } \\
\text { igualitário do grupo GC. A FCR, do } \\
\text { grupo GRV, quando analisada, se } \\
\text { mostrou superior (p<0,05) ao limite } \\
\text { esperado, se mantendo em níveis } \\
\text { elevados até cinco minutos após a } \\
\text { atividade. }\end{array}$ & $\begin{array}{l}\text { Não } \\
\text { relatado }\end{array}$ \\
\hline $\begin{array}{l}\text { Cacau et } \\
\text { al., } 2013\end{array}$ & $\begin{array}{l}\text { Avaliar o uso da RV na } \\
\text { reabilitação funcional de } \\
\text { pacientes pós-cirurgia } \\
\text { cardíaca. }\end{array}$ & $\begin{array}{c}\mathrm{n}=60 \\
\mathrm{GC} \mathrm{n}=30 \\
(\mathrm{~F}=17 / \mathrm{M}=13) \\
\text { GRV } \mathrm{n}=30(\mathrm{~F}=14 / \\
\mathrm{M}=16) \\
\text { Média de idade: } \\
\text { 50,60 anos }\end{array}$ & $\begin{array}{l}\text { MIF, TC6, } \\
\text { Nottingham } \\
\text { Health Profile } \\
\text { (NHP) }\end{array}$ & $\begin{array}{c}\text { Não } \\
\text { descrito }\end{array}$ & $\begin{array}{l}\text { 2x/dia } \\
\text { Hospitalar }\end{array}$ & $\begin{array}{l}\text { A MIF apresentou diferença } \\
\text { significativa apenas no escore motor } \\
\text { e total no } 1^{\circ} \text { PO }(p<0,05) \\
\text { intragrupos. O NHP não apresentou } \\
\text { nenhuma diferença no escore total } \\
\text { em ambos os grupos avaliados } \\
(\mathrm{p}<0,05) \text {. No domínio do GRV } \\
\text { apresentou redução da intensidade } \\
\text { desse sintoma quando comparado ao } \\
\text { GC (p<0,05) no } 3^{\circ} \mathrm{PO} \text {. Houve } \\
\text { aumento no nível de energia no } 1^{\circ} \\
\text { PO (p<0,05), associado a uma } \\
\text { redução no tempo de internamento } \\
(\mathrm{p}<0.05) \text { e aumento da } \mathrm{DP}(\mathrm{p}<0.05) .\end{array}$ & $\begin{array}{l}\text { Não } \\
\text { relatado }\end{array}$ \\
\hline $\begin{array}{l}\text { Rutkowski } \\
\text { et al., } \\
2020\end{array}$ & $\begin{array}{l}\text { Comparar os efeitos do } \\
\text { programa de reabilitação } \\
\text { baseado em pacientes } \\
\text { internados com DPOC } \\
\text { utilizando a RV não } \\
\text { imersiva no treinamento } \\
\text { em um programa de } \\
\text { reabilitação pulmonar. }\end{array}$ & $\begin{array}{c}\mathrm{n}=110 \\
\mathrm{TE}=\mathrm{n} 34 \\
(\mathrm{~F}=16 / \mathrm{M}=18) \\
\mathrm{TE}+\mathrm{RV} \mathrm{n}=38 \\
(\mathrm{~F}=19 / \mathrm{M}=19) \\
\mathrm{RV} \mathrm{n}=38 \\
(\mathrm{~F}=28 / \mathrm{M}=10) \\
\\
\text { Média de idade: } \\
61,03 \text { anos }\end{array}$ & $\begin{array}{c}\text { Teste Fitness } \\
\text { Sênior }\end{array}$ & $\begin{array}{c}\text { Xbox } \\
360^{\circledR} \\
\text { Kinect }^{\circledR}\end{array}$ & $\begin{array}{l}20 \text { min } \\
5 \\
\text { dias/semanas } \\
2 \text { semanas } \\
\text { Hospitalar }\end{array}$ & $\begin{array}{l}\text { Na análise intergrupos, o grupo } \\
\text { TE+RV apresentou melhora em } \\
\text { todos os componentes do Teste } \\
\text { Fitness Sênior }(p<0,05) \text {, No RV e } \\
\text { TE+RV, houve aumento da DP } \\
(p<0,05) \text {. }\end{array}$ & $\begin{array}{l}\text { Não } \\
\text { relatado }\end{array}$ \\
\hline $\begin{array}{l}\text { Lima et } \\
\text { al., } \\
2020\end{array}$ & $\begin{array}{l}\text { Avaliar o efeito da RV na } \\
\text { função pulmonar e } \\
\text { independência funcional } \\
\text { em pacientes submetidos a } \\
\text { revascularização do } \\
\text { miocárdio }\end{array}$ & $\begin{array}{c}\mathrm{n}=56 \\
\mathrm{GC} \mathrm{n}=25 \\
(\mathrm{~F}=8 / \mathrm{M}=17) \\
\text { GRV } \mathrm{n}=31(\mathrm{~F}: 10 / \\
\mathrm{M}: 21) \\
\text { Média de idade: } \\
\text { 52,50 anos }\end{array}$ & $\begin{array}{c}\text { PImáx, PEmáx, } \\
\text { PFE, CV, } \\
\text { TUG, MIF }\end{array}$ & $\begin{array}{c}\text { Xbox } \\
360^{\circledR} \\
\text { Kinect }^{\circledR}\end{array}$ & $\begin{array}{l}20 \mathrm{~min} \\
2 \mathrm{x} / \text { dia } \\
5 \text { dias } \\
\text { Hospitalar }\end{array}$ & $\begin{array}{l}\text { Na análise intergrupos, a PImáx } \\
\text { final, PEmáx final, o grupo GRV } \\
\text { apresentaram } \\
\text { significativos }(\mathrm{p}<0,05) \text {. O VC e o } \\
\text { PFE não obtiveram diferença } \\
\text { significativa }(\mathrm{p}>0,05) \text {. No escore da } \\
\text { MIF, o GRV apresentou melhora } \\
\text { da funcionalidade }(\mathrm{p}<0,05) \text {. O tempo } \\
\text { do TUG, do GRV reduziu em } \\
\text { comparação ao GC }(\mathrm{p}<0,05) \text {. }\end{array}$ & $\begin{array}{l}\text { Não } \\
\text { relatado }\end{array}$ \\
\hline
\end{tabular}

CV: Capacidade Vital, DP: distância percorrida, DPOC: doença pulmonar obstrutiva crônica, F: feminino, FC: frequência Cardíaca, FR: frequência respiratória, FCR: frequência cardíaca de reserva, GC: grupo controle, GRV: grupo realidade virtual, IMC: índice de massa corporal, M: masculino, MIF: Medida de Independência Funcional, NHP: Nottingham Health Profile, PAD: pressão arterial diastólica, PAS: pressão arterial sistólica, PEmáx: pressão expiratória máxima, PFE: fluxo expiratório máximo, PImáx: pressão inspiratória máxima, PO: pósoperatório, $\mathrm{RV}$ : realidade virtual, $\mathrm{SpO}_{2}$ : saturação periférica de oxigênio, TC6: Teste de Caminhada de seis minutos, TE: treino apenas de exercícios, TE+RV: treino de exercícios mais realidade virtual, TUG: Time up to Go, VC: volume corrente. Fonte: Autores. 


\section{Discussão}

A fisioterapia cardiorrespiratória vem testemunhando a incorporação de recursos tecnológicos ao plano terapêutico. A RV é um destes recursos e seu uso já tem demonstrado benefícios em diversas áreas da fisioterapia (Silva et al. 2021; Tefertiller et al., 2019; Elliot et al., 2015; Yelvar et al. 2017; Feyzioglu et al. 2020), pois impulsiona a interação dos pacientes com a terapia e incentiva o protagonismo no processo de recuperação (Silva et al. 2018), o que resulta na melhora da funcionalidade e resistência ao exercício (Cacau et al. 2013). Desta forma, evidências de impacto moderado e alto (Carvalho, Silva \& Grande, 2013) apoiam a utilização da RV como método coadjuvante na fisioterapia cardiorrespiratória.

Para a avaliação da capacidade cardiorrespiratória e resistência aos exercícios, três artigos (Silva et al. 2018; Rutkowski et al. 2020; Cacau et al., 2013) utilizaram o TC6 como parâmetro. Evidências moderadas e de baixo risco de viés mostraram uma melhora significativa na distância percorrida nos grupos que utilizaram à RV simultânea ao exercício físico, em comparação ao grupo controle (GC) durante o período de tratamento. Esse aumento da distância percorrida no TC6 pode ser justificado pelo aumento do consumo máximo de oxigênio durante a atividade, como resposta pelo estímulo visual, por meio da RV e pelas adaptações induzidas pelo exercício aeróbico gerando efeitos hemodinâmicos, como elevação da FR, FC e pressão arterial (PA) (Silva et al., 2018).

Apenas um estudo considerou os efeitos hemodinâmicos (Alves da Cruz et al. 2020), mostrando que os pacientes submetidos à RV promoveram maior intensidade na frequência cardíaca (FC) de reserva e na PA sistólica, nos últimos minutos de terapia, quando comparado ao GC. No entanto, nenhuma diferença estatística foi sugestiva de maior intensidade do esforço, além de apresentar maior repercussão hemodinâmica até cinco minutos após a interrupção do exercício no período de recuperação. Os valores maiores de PA sistólica encontrados no grupo realidade virtual (GRV) podem ser justificados em decorrência da redução abrupta da intensidade do exercício. Além disso, a maior intensidade de FC de reserva pode estar relacionada com o aumento da FC, sugerindo um maior esforço na atividade gerada pela RV, podendo estar associada à complexidade das atividades solicitadas e pelo fato de a terapia ser realizada aos pares, instigando a competitividade entre os pacientes (Lima et al. 2020).

Cacau et al. (2013) relataram notória perda funcional, tanto no GRV quanto no CG, pois os pacientes estudados foram submetidos a cirurgias de grande porte como, revascularização do miocárdio e troca valvar. A redução da funcionalidade é esperada, por se tratar de pacientes submetidos a procedimentos cirúrgicos que necessitam de cuidados intensivos, com o uso de dispositivos que reduzem a mobilidade no leito, com consequente perda de massa muscular (Cordeiro et al., 2016), além da dor na região cirúrgica (Orcutt et al., 2012). Entretanto o GRV evoluiu com menor queda no desempenho funcional. Justificase esse achado pela análise do domínio do nível de energia, avaliado pelo Nottingham Health Profile, que observou maior nível de energia no GRV quando comparado com o GC, visto que, quanto mais energia o paciente apresentar, melhor será a resposta aos exercícios propostos durante a terapia. Este aumento de energia pode ser secundário a motivação e interação do paciente que a RV proporciona, tanto Cacau et al. (2013) quanto Lima et al. (2020) descreveram melhora gradual na recuperação física e funcional, ainda em ambiente hospitalar, baseadas na escala Medida de Independência Funcional.

Outro fator importante explorado por Cacau et al. (2013) foi a dor, mensurado pelo Nottingham Health Profile. Os autores descreveram redução significativa deste sintoma no GRV quando comparado ao GC, visto que nenhum grupo foi submetido a recurso fisioterapêutico analgésico e todos permaneciam com o protocolo de analgesia padrão.

O uso da RV no tratamento fisioterapêutico resultou em menor redução das forças musculares respiratórias, tanto inspiratória, quanto expiratória, em pacientes submetidos a revascularização do miocárdio (LIMA et al. 2020). Pacientes submetidos a cirurgias cardíacas, podem evoluir com alterações na mecânica respiratória em decorrência dos traumas na linha média, acarretando mudanças no padrão respiratório e redução das pressões respiratórias (PImax e PEmax) (Vanderline, 2010), 
portanto, o uso da RV como coadjuvante no tratamento fisioterapêutico parece ser promissor.

Além de redução da dor, melhora da funcionalidade e minimizar a perda de força muscular respiratória, a VR parece reduzir o tempo de internação dos pacientes cardiopatas (Cacau et al. 2013; Lima et al. 2020). Outro achado relatado na literatura analisada (Silva et al., 2018) foi ausência de resultados relevantes no índice glicêmico.

Em resumo, os estudos incluídos defendem que a interação com o mundo virtual redireciona a atenção ao objetivo do jogo ou à tarefa a ser executada, minimizando o foco em relação ao fator limitante. Entretanto a heterogeneidade da amostra, dos objetivos e metodológicas, principalmente entre os instrumentos de avaliação, nos impediram de realizar análise estatística dos resultados dos estudos, para determinação quantitativa dos efeitos da RV sobre a capacidade cardiorrespiratória dos pacientes. A ausência dessa análise limita as afirmações mais assertivas em relação aos tipos de RV utilizar, protocolos e frequência de uso nessa população.

\section{Considerações Finais}

$\mathrm{O}$ uso da RV no tratamento fisioterapêutico cardiorrespiratório apresenta resultados semelhantes ao tratamento convencional, na melhora da capacidade funcional e física, com benefícios motores e respiratórios. Quando o objetivo é estimular o paciente durante o tratamento fisioterapêutico, seu uso torna-se um recurso adicional. Ressalta-se a necessidade de mais estudos na área e sua padronização para uma análise mais robusta dos resultados.

\section{Referências}

Almeida, P., \& Rodrigues, F. (2014). Exercise training modalities and strategies to improve exercise performance in patients with respiratory disease. Revista portuguesa de pneumologia, 20(1), 36-41.

Bernard, S., Ribeiro, F., Maltais, F., \& Saey, D. (2014). Prescribing exercise training in pulmonary rehabilitation: a clinical experience. Revista portuguesa de pneumologia, 20(2), 92-100.

Burdea, G C. (2003). Reabilitação virtual - benefícios e desafios. Métodos de Informação em Medicina, 42(05), 519-523.

Cacau, L., Oliveira, G. U., Maynard, L. G., Araújo Filho, A. A., Silva, W. M., Jr, Cerqueria Neto, M. L., Antoniolli, A. R., \& Santana-Filho, V. J. (2013). The use of the virtual reality as intervention tool in the postoperative of cardiac surgery. Revista brasileira de cirurgia cardiovascular: órgão oficial da Sociedade Brasileira de Cirurgia Cardiovascular, 28(2), 281-289.

Caiana, T. L, Nogueira, D. L, \& Lima, A. C. D. (2016). A realidade virtual e seu uso como recurso terapêutico ocupacional: revisão integrativa. Cadernos de Terapia Ocupacional da Universidade Federal de São Carlos, 24 (3),575-589.

Cordeiro, A. L., de Melo, T. A., Neves, D., Luna, J., Esquivel, M. S., Guimarães, A. R., Borges, D. L., \& Petto, J. (2016). Inspiratory Muscle Training and Functional Capacity in Patients Undergoing Cardiac Surgery. Brazilian journal of cardiovascular surgery, 31(2), $140-144$.

Carvalho, A., Silva, V., \& Grande, A. J. (2013). Avaliação do risco de viés de ensaios clínicos randomizados pela ferramenta da colaboração Cochrane. Diagnóstico Tratamento, 18(1), 38-44.

Carvalho, J. A. (2012) Virtual reality and ophthalmology. Revista Brasileira de Oftalmologia, 71(1), 40-47.

Alves da Cruz, M. M., Ricci-Vitor, A. L., Bonini Borges, G. L., Fernanda da Silva, P., Ribeiro, F., \& Marques Vanderlei, L. C. (2020). Acute Hemodynamic Effects of Virtual Reality-Based Therapy in Patients of Cardiovascular Rehabilitation: A Cluster Randomized Crossover Trial. Archives of physical medicine and rehabilitation, 101(4), 642-649.

Elliott, V., de Bruin, E. D., \& Dumoulin, C. (2015). Virtual reality rehabilitation as a treatment approach for older women with mixed urinary incontinence: a feasibility study. Neurourology and urodynamics, 34(3), 236-243.

Feyzioğlu, Ö., Dinçer, S., Akan, A., \& Algun, Z. C. (2020). Is Xbox 360 Kinect-based virtual reality training as effective as standard physiotherapy in patients undergoing breast cancer surgery. Supportive care in cancer: official journal of the Multinational Association of Supportive Care in Cancer, 28(9), 42954303 .

Galvão, T. F, Pansani, T. S. A, \& Harrad, D. (2015). Principais itens para relatar Revisões sistemáticas e Meta-análises: A recomendação PRISMA. Epidemiologia e Serviços de Saúde, 24, 335-342.

Lima, H. J, Souza, R. S. P, Santos, A.S.M. Borges, D. L, Guimarães, A.R. F Ferreira, G. V. D. B. A. \& Cordeiro, A. L. L. (2020). Realidade virtual da função pulmonar e independência funcional após cirurgia de revascularização do miocárdio: ensaio clínico. American Journal of Cardiovascular Disease, 10(4),499. 
Research, Society and Development, v. 10, n. 17, e209101724711, 2021 (CC BY 4.0) | ISSN 2525-3409 | DOI: http://dx.doi.org/10.33448/rsd-v10i17.24711

Okasheh, R., Al-Yahya, E., Al-Khlaifat, L., Almasri, N., Muhaidat, J., \& Qutishat, D. (2019). Advancing Cardiorespiratory Physiotherapy Practice in a Developing Country: Surveying and Benchmarking. Rehabilitation research and practice, 2019, 7682952.

Oliveira J. G. D, Souza, M.E, Coutinho, S.M.A, Castro, S, Paulo, I.M.M. (2015) Use of associated electrotherapy kinesiotherapy in patients with osteoarthrritis treatment. Fisioterapia Ser, 10(2).

Orcutt, S. T., Bechara, C. F., Pisimisis, G., Barshes, N. R., \& Kougias, P. (2012). Impact of perioperative events on mortality after major vascular surgery in a veteran patient population. American journal of surgery, 204(5), 586-590.

Rutkowski, S., Rutkowska, A., Kiper, P., Jastrzebski, D., Racheniuk, H., Turolla, A., Szczegielniak, J., \& Casaburi, R. (2020). Virtual Reality Rehabilitation in Patients with Chronic Obstructive Pulmonary Disease: A Randomized Controlled Trial. International journal of chronic obstructive pulmonary disease, 15, $117-124$

da Costa Santos, C. M., de Mattos Pimenta, C. A., \& Nobre, M. R. (2007). The PICO strategy for the research question construction and evidence search. Revista latino-americana de enfermagem, 15(3), 508-511.

Silva, J. P. L. N.S, Novaes, L.F.M, Santos, L.C.R, Galinho, B.P, Calcante, M.A, Araujo, B.C.G. ... Freire. A. P. C. F. (2018). Effects of Conventional and Virtual Reality Cardiovascular Rehabilitation in Body Composition and Functional Capacity of Patients with Heart Diseases: Randomized Clinical Trial, International Journal of Cardiovascular Sciences, 31, 619-629.

da Silva, T. D., de Oliveira, P. M., Dionizio, J. B., de Santana, A. P., Bahadori, S., Dias, E. D., Ribeiro, C. M., Gomes, R. A., Ferreira, M., Ferreira, C., de Moraes, I., Silva, D., Barnabé, V., de Araújo, L. V., Santana, H., \& Monteiro, C. (2021). Comparison Between Conventional Intervention and Non-immersive Virtual Reality in the Rehabilitation of Individuals in an Inpatient Unit for the Treatment of COVID-19: A Study Protocol for a Randomized Controlled Crossover Trial. Frontiers in psychology, 12, 622618.

Tanović, E., Talić-Tanović, A., Đelilović-Vranić, J., Rekić, A., Papović, A., \& Tanović, E. (2019). Effects of kinesiotherapy on muscle strengthening in patients with Parkinson disease. Medicinski glasnik :official publication of the Medical Association of Zenica-Doboj Canton, Bosnia and Herzegovina, 16(1), 10.17392/970-18. Advance online publication.

Tefertiller, C., Hays, K., Natale, A., O'Dell, D., Ketchum, J., Sevigny, M., Eagye, C. B., Philippus, A., \& Harrison-Felix, C. (2019). Results From a Randomized Controlled Trial to Address Balance Deficits After Traumatic Brain Injury. Archives of physical medicine and rehabilitation, 100(8), $1409-1416$.

Vandrline, F. (2010). Vídeo games na saúde e reabilitação. Editora Schoba.

Yilmaz Yelvar, G. D., Çırak, Y., Dalkılınç, M., Parlak Demir, Y., Guner, Z., \& Boydak, A. (2017). Is physiotherapy integrated virtual walking effective on pain, function, and kinesiophobia in patients with non-specific low-back pain? Randomised controlled trial. European spine journal: official publication of the European Spine Society, the European Spinal Deformity Society, and the European Section of the Cervical Spine Research Society, $26(2), 538-545$. 